\title{
Effects of the rotavirus vaccine program across age groups in the United States: analysis of national claims data, 2001-2016
}

\author{
Julia M. Baker ${ }^{1,2^{*}}$ (D), Rebecca M. Dahl', Justin Cubilo1, Umesh D. Parashar ${ }^{2}$ and Benjamin A. Lopman ${ }^{1,2}$
}

\begin{abstract}
Background: The direct effectiveness of infant rotavirus vaccination implemented in 2006 in the United States has been evaluated extensively, however, understanding of population-level vaccine effectiveness (VE) is still incomplete.

Methods: We analyzed time series data on rotavirus gastroenteritis (RVGE) and all-cause acute gastroenteritis (AGE) hospitalization rates in the United States from the MarketScan ${ }^{\oplus}$ Research Databases for July 2001-June 2016. Individuals were grouped into ages 0-4, 5-9, 10-14, 15-24, 25-44, and 45-64 years. Negative binomial regression models were fitted to monthly RVGE and AGE data to estimate the direct, indirect, overall, and total VE.

Results: A total of 9211 RVGE and 726,528 AGE hospitalizations were analyzed. Children 0-4 years of age had the largest declines in RVGE hospitalizations with direct VE of 87\% (95\% Cl: 83, 90\%). Substantial indirect effects were observed across age groups and generally declined in each older group. Overall VE against RVGE hospitalizations for all ages combined was 69\% (95\% Cl: 62, 76\%). Total VE was highest among young children; a vaccinated child in the post-vaccine era has a 95\% reduced risk of RVGE hospitalization compared to a child in the pre-vaccine era. We observed higher direct VE in odd post-vaccine years and an opposite pattern for indirect VE.
\end{abstract}

Conclusions: Vaccine benefits extended to unvaccinated individuals in all age groups, suggesting infants are important drivers of disease transmission across the population. Imperfect disease classification and changing disease incidence may lead to bias in observed direct VE.

Trial registration: Not applicable.

Keywords: Rotavirus, Gastroenteritis, Vaccination, Hospitalization, United States

\section{Background}

The United States (US) was one of the first countries to introduce infant rotavirus vaccination nationally [1] and dramatic changes in the rotavirus disease burden and epidemiologic patterns of diarrheal disease have followed [2]. Prior to vaccine introduction in 2006, [3] rotavirus was estimated to cause 55,000-70,000 hospitalizations and over 600,000 emergency room and outpatient/office visits among children under 5 years of age in the US annually $[3,4]$. Consistent annual peaks in disease incidence

\footnotetext{
* Correspondence: julia.baker@emory.edu

'Department of Epidemiology, Rollins School of Public Health, Emory University, 1518 Clifton Road NE, Atlanta, GA 30322, USA

${ }^{2}$ Division of Viral Diseases, National Center for Immunization and Respiratory Diseases, Centers for Disease Control and Prevention, 1600 Clifton Road NE, Atlanta, GA 30333, USA

Full list of author information is available at the end of the article
}

occurred in winter and early spring [4]. Early evaluations of rotavirus seasonality in the post-vaccine era identified substantial alterations of disease patterns, including a reduced magnitude, delayed onset, and shorter duration of the rotavirus season [2]. Further, there has been a distinct shift from annual to biennial peaks in disease incidence among children under 5 years of age, $[2,5,6]$ a pattern not observed in some other high-income countries that have introduced the vaccine [7-9].

The direct vaccine effectiveness (VE) of rotavirus vaccine has been evaluated extensively while understanding of indirect vaccine effects is still incomplete. Substantial vaccine impacts are evidenced by $50-90 \%$ reductions in rotavirus hospitalizations among young, vaccine-eligible children [10]. A recent meta-analysis estimated a direct $\mathrm{VE}$ of $84 \%$ against rotavirus-associated hospitalizations

(c) The Author(s). 2019 Open Access This article is distributed under the terms of the Creative Commons Attribution 4.0 International License (http://creativecommons.org/licenses/by/4.0/), which permits unrestricted use, distribution, and 
or emergency department visits in the US [11]. Notably, this estimate is limited by imperfect rotavirus diagnostics [12] largely due to incomplete testing for rotavirus in the clinical setting [13]. In addition to the remarkable direct effects, reductions in rotavirus disease have exceeded vaccine coverage, suggesting indirect benefits to unvaccinated children [6]. These indirect benefits may extend to children too young to be vaccinated, age-ineligible older children, adolescents, and adults among whom reductions in rotavirus gastroenteritis (RVGE) and all-cause acute gastroenteritis (AGE) have been observed [14].

The long-term impact of a vaccine program will be governed by the direct effects of vaccinating children together with the transmission-modulating consequences of vaccination as described by Halloran et al. [15] Theoretically, direct effects, which represent the biological protection obtained from vaccination at the individual level, [16] are a vaccine characteristic that remains constant over time (except with waning immunity) and are independent of vaccine coverage. Conversely, population-level effects can vary with changes in vaccine coverage, population immunity, and social mixing patterns $[15,17,18]$. These population-level effects include (a) indirect effects or "herd protection" provided to unvaccinated individuals, (b) total effects which describe the combination of biologic and indirect protection received by vaccinated individuals, and (c) the overall effects which quantify the public health benefit of a vaccination program by weighting the total effects among the vaccinated and indirect effects among the unvaccinated populations $[15,19]$.

Given the relative novelty of the rotavirus vaccine in the US, there have been few evaluations of how vaccine effects may change during the post-vaccine era and their relationship with disease patterns. In order to quantify the full, population-wide impacts of infant rotavirus vaccination, longer-term evaluations of vaccine effects across age groups are needed. Understanding these phenomena could lead to strategies that maximize the program's benefits and anticipate future healthcare resource needs (e.g. biennial versus annual epidemics). This study aimed to quantify the direct, indirect, overall, and total effectiveness of infant rotavirus vaccination on hospitalization for RVGE and AGE across age groups and their annual variation during the post-vaccine era in the US.

\section{Methods}

\section{Data source and study period}

We analyzed data from the $\mathrm{IBM}^{\circ}$ MarketScan ${ }^{\circ}$ Commercial Database, a collection of national medical claims and encounters data from commercially insured individuals under 65 years of age in the US. The database includes de-identified, individual-level enrollment, inpatient, and outpatient medical data on employees, their spouses and dependents with employer-sponsored health care insurance in all US states. This encompasses a variety of health plans such as PPOs, POS plans and HMOs but does not include claims covered by Medicaid. The database contains information on several million individuals each year [20].

We analyzed time series data on monthly RVGE and AGE hospitalization rates for July 2001-June 2016. Study years were defined from July through June of the following calendar year and identified by the year in which the rotavirus season occurred (e.g. July 2007-June 2008 was identified as "2008”).

\section{Identification of RVGE and AGE hospitalizations}

Monthly counts of RVGE hospitalizations included individuals with a rotavirus International Classification of Diseases, Ninth and Tenth Revision (ICD-9 and ICD-10) code (008.61 and A08.0, respectively). Given the incomplete detection of rotavirus using ICD coding, [12] data on AGE was also compiled in order to represent possible RVGE not identified as rotavirus-related. Applicable ICD-9/10 codes for AGE include bacterial, parasitic, and viral gastrointestinal illness of determined etiology and presumed infectious or non-infectious gastrointestinal illness of undetermined etiology [21]. For both RVGE and AGE, the ICD-9/10 codes were identified in one of 15 diagnosis fields from inpatient admission claims.

All RVGE and AGE inpatient claims among children, adolescents, and adults not age-eligible to receive the rotavirus vaccine during the study period were included in the analysis and were considered unvaccinated. For children less than 10 years of age who were age-eligible for the vaccine, only those who were continuously enrolled from birth through 6 months of age were included in the analysis. This continuous enrollment requirement aimed to reduce misclassification of vaccination status by helping ensure that rotavirus vaccination occurring within the $\mathrm{CDC}$ recommended schedule (at 2, 4 and 6 months of age) [22] was captured in the insurance claim records. Children age-eligible for vaccination but without continuous follow-up were excluded from the analysis because of their unknown vaccination status.

Individuals were grouped into ages $0-4,5-9,10-14$, $15-24,25-44$, and $45-64$ years (data on adults aged 65 years and older are not available in the MarketScan ${ }^{\circ}$ Commercial Database and were therefore excluded). Children under 5 were additionally categorized into one-year age groups and children under 10 were stratified by vaccination status. The number of enrollment member days were summed by month, year, age group, and vaccination status to provide the enrolled population denominator for each month of the study period and enable calculation of rates. 


\section{Vaccination status}

For this analysis, child vaccination status was tracked beginning in July 2006 when the first cohort of newborns became age-eligible for vaccination the following month, coinciding with the Centers for Disease Control and Prevention Advisory Committee on Immunization Practices' August announcement [3] recommending the vaccine. Children who received at least one dose of either available rotavirus vaccine, RotaTeq (RV5) or Rotarix (RV1) were considered vaccinated. Current Procedural Terminology (CPT) was used to define receipt of RV5 or RV1 based on CPT codes 90680 and 90681, respectively. In order to further reduce misclassification of vaccination status, all individuals (children and adults) residing in states with universal vaccine purchasing programs, which provide immunizations to children free of charge, were excluded from the analysis throughout the study period as vaccination in these states may not be recorded in insurance claim records (Alaska, Connecticut, Idaho, Massachusetts, Maine, North Dakota, New Hampshire, New Mexico, Oregon, Rhode Island, South Dakota, Vermont, Washington, Wisconsin, and Wyoming).

\section{Statistical analysis}

Negative binomial regression models were fitted to monthly RVGE and AGE count data to estimate rate ratios (RR) and 95\% confidence intervals (CIs) from which vaccine effects were calculated. Negative binomial models were chosen after overdispersion was identified in preliminary models using Poisson regression. Using the framework proposed by Halloran, [15] we estimated the (a) direct effectiveness of the vaccine by comparing the rates in vaccinated and unvaccinated groups in the post-vaccine era, (b) indirect effectiveness by comparing rates among unvaccinated groups in the post-vaccine era to the pre-vaccine era, (c) overall effectiveness by comparing average rates in the post- and pre-vaccine eras, and (d) total effectiveness by comparing rates in the vaccinated groups in the post-vaccine era to the corresponding groups (all unvaccinated) in the pre-vaccine era. The count of RVGE and AGE cases was modeled using the glm.nb package in $\mathrm{R}$ with adjustment for changes in person-years of follow-up using an offset of the log of person-years. Each model included one dichotomous predictor to differentiate the comparison groups: vaccination status (direct), pre- vs. post-vaccine era (indirect and overall), and vaccinated and post-vaccine era vs. pre-vaccine era (total). VE was calculated as 1-RR.

Models were fitted separately for each age group. Direct and total VE was estimated for all age groups eligible for rotavirus vaccination by the end of the study period, with children under 1 year of age vaccine eligible during the entire post-vaccine period (July 2007-June 2016) and children 9 years of age only eligible during the last year of study data (July 2015-June 2016). Indirect and overall VE were estimated for all age groups, though these values were equal for groups ineligible for vaccination throughout the study period because vaccination coverage equaled zero. For the 0-4 age group, direct, indirect, total, and overall effects were additionally calculated for each individual post-vaccine year beginning in 2008 to estimate annual changes in VE. For all age groups, indirect VE was estimated for individual post-vaccine years.

RVGE models were fitted using full-year data. To improve model specificity, AGE models were restricted to the historic rotavirus season of January-June. The year immediately following vaccine introduction, July 2006June 2007, was excluded as a transition period for all models. The inclusion of a continuous time variable was considered for all models in an effort to adjust for potential secular trends unrelated to vaccination that may have impacted rates. None of the RVGE model results were sensitive to the addition of the time variable based on Akaike information criterion (AIC) and the variable was therefore excluded. Analyses were performed using R software.

\section{Investigation into annual variation in direct VE}

Preliminary estimates of direct vaccine effects among the $0-4$ age group appeared to vary annually. Given that direct VE should not change over time (in the absence of waning), we performed calculations to evaluate whether this observed variation could be explained by the combination of imperfect coding of rotavirus and annual variation in disease incidence leading to different magnitudes of misclassification of cases/non-cases in post-vaccine years. In other words, even with constant sensitivity and specificity of ICD-9/10 codes, the number of rotavirus positive/negative cases that are misclassified (and which are ultimately used in calculations of VE) will vary based on the incidence of disease. In years with higher disease incidence, there may be a larger number of individuals misclassified and vice versa. We began with a hypothetical population and applied input parameters of true VE, vaccine coverage, and rotavirus incidence in the unvaccinated population from 2010 to 2016; this enabled estimation of the number of 'true' RVGE cases among the vaccinated and unvaccinated children. We then applied realistic values for rotavirus ICD-9 code sensitivity (0.5) and specificity (0.99) [12] to this 'true' data to estimate 'projected' RVGE cases. From these values, we calculated a 'projected' VE and compared this to our 'observed' VE estimated via regression analysis and 'true' VE (an input parameter).

\section{Role of the funding source}

This study received no dedicated funding. The corresponding author had full access to all data in the study 
and had final responsibility for the decision to submit for publication.

\section{Results}

From July 2001-June 2016, there were a total of 9211 RVGE hospitalizations and 726,528 AGE hospitalizations across all age groups (Additional file 1: Table S1). Over 91\% of RVGE hospitalizations (91.4\%) and over half of AGE hospitalizations (54.0\%) occurred during the rotavirus season of January-June.

\section{RVGE time series}

\section{Young children, 0-9 years of age}

Among children 0-9 years of age, the pre-vaccine period displayed a consistent pattern of rotavirus illness with single annual peaks in hospitalization rates during the winter/spring months (Figs. 1 and 2). Rates declined dramatically for child age groups after introduction of the vaccine and both the 0-4 and 5-9 groups settled into a biennial pattern with the highest rates observed in odd post-vaccine years. When the 0-4 group was further examined by single year and vaccinated/unvaccinated cohorts, the biennial pattern became more apparent among the unvaccinated and younger children. Among the vaccinated children, the biennial pattern was clearest among those 2 years of age and younger. Notably, rates among older, unvaccinated children returned to levels similar to those seen prior to vaccine introduction after initial declines in the post-vaccine period. In contrast, the rates of RVGE remained dramatically lower in the post-vaccine period among vaccinated children.

\section{Older children, adolescents and adults}

RVGE rates among older children, adolescents, and adults during the pre-vaccine era did not display the same distinctive pattern observed among young children (Fig. 2). Rather, the pre-vaccine period for these groups was characterized by frequent, irregular spikes in rates. After vaccine introduction, the sporadic pattern continued, however, at a lower rate and now punctuated with biennial peaks corresponding to those seen among young children.

\section{RVGE VE for entire post-vaccine period}

The largest declines in RVGE hospitalizations were observed among the youngest children (Table 1). Direct VE was $87 \%$ (95\% CI: 83, 90\%) among children 0-4 years of age.

Substantial indirect effects were observed across age groups and these effects generally declined in each older group. Indirect VE against RVGE hospitalization among unvaccinated children under 1 year of age was $79 \%$ (95\% CI: 66, 87\%) compared to adults 45-64 years of age among whom indirect VE was 35\% (95\% CI: 9, 53\%).
One exception to this general trend was the greater indirect VE in adults 25-44 years of age (indirect VE: $56 \%$; 95\% CI: $36,70 \%)$.

Overall VE against RVGE hospitalizations for the entire study population (all ages) combined was $69 \%$ (95\% CI: 62, $76 \%$ ). Significant reductions in hospitalization rates were observed across all ages and the overall vaccine effectiveness generally declined in each older group (Table 1).

Total vaccine effects mirrored the pattern seen in direct and indirect effects, with the largest total VE observed in the youngest children (Table 1, total VE for children 0-4 years of age: $95,95 \%$ CI: $93,96 \%$ ).

\section{RVGE direct and indirect VE by post-vaccine year}

After relatively consistent direct VE immediately following vaccine introduction, we observed a possible alternating pattern in estimated direct VE among children $0-4$ years of age, with the slightly higher direct VE in odd post-vaccine years (Fig. 3). A more extreme and opposite pattern was apparent for indirect VE for this group, with higher indirect VE during even post-vaccine years compared to odd post-vaccine years. This pattern extended to all ages (Fig. 4). Both VE measures displayed wide CIs due to small numbers of cases.

Calculations to evaluate how variations in disease incidence impacted direct VE revealed a pattern in projected direct VE similar to that of our observed direct VE (Table 2). Our calculations used a population of $1,000,000$, vaccine coverage of $50 \%$, true VE of $95 \%$, sensitivity of 0.5 , specificity of 0.99 , and estimated RVGE incidence among unvaccinated children for each year from 2010 to 2016 from the MarketScan ${ }^{\circ}$ Commercial Database.

\section{AGE time series}

The observations for RVGE rates among children were consistent with those observed for AGE rates in the same population, though the patterns were often less distinct. The pre-vaccine era was characterized by consistent, annual peaks that shifted towards a biennial pattern in the post vaccine era among young children; the biennial patterns were most apparent among the youngest and the unvaccinated children (Fig. 5). Among older children, adolescents, and adults, the pre-vaccine era displayed relatively erratic patterns in AGE rates (Additional file 1: Figure S1). Unlike RVGE rates, the post-vaccine period was not punctuated by clear biennial peaks in these age groups and a slight increasing trend in rates was observed among those age 10 and older.

\section{AGE VE for entire post-vaccine period}

Significant direct, indirect, overall, and total VE was observed among both $0-4$ and 5-9year age groups with the largest impacts among the youngest children (Table 3). Among older age groups, VE estimates were highly sensitive 

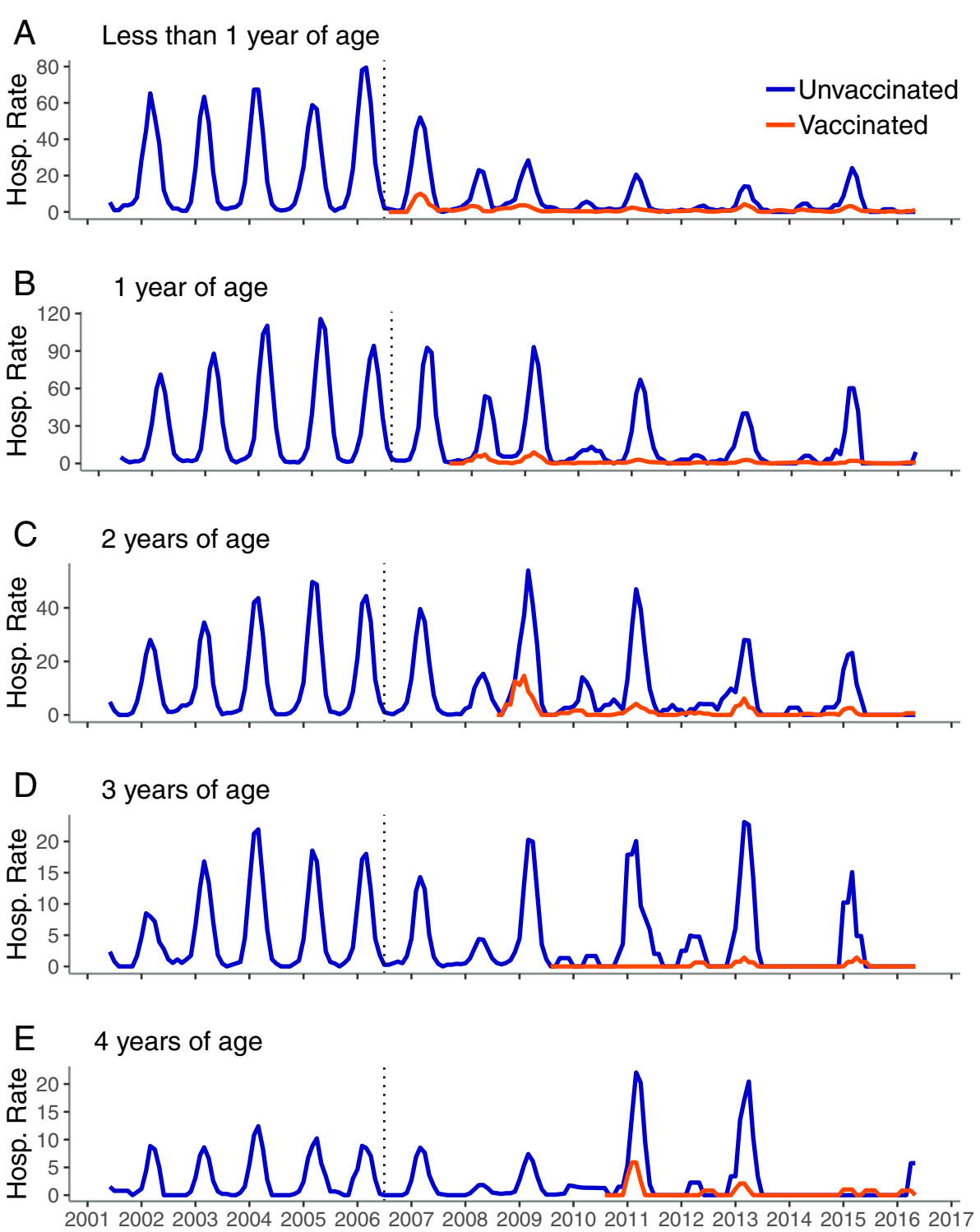

Fig. 1 Monthly inpatient RVGE rates per 10,000 person-years, United States, July 2001-June 2016, children 0-4 years. ${ }^{\text {a }}$ Legend: a: less than 1 year of age, $\mathbf{b}$ : 1 year of age, c: 2 years of age, $\mathbf{d}$ : 3 years of age, e: 4 years of age ${ }^{\text {a }}$ Time series includes all years (including 2007 transition year) and all months (not restricted to the historic rotavirus season), Vertical dashed line represents July 2006 (time of vaccine introduction)

to the method used to control for time. Several methods were considered, including use of a continuous time variable as well as higher order terms. Estimates of VE and their statistical significance varied based on the method used, however, no single model was found to be markedly superior to the rest based on AIC values. Given the uncertainty of these models and output, these results are not presented.

\section{Discussion}

Vaccines may have impacts that go beyond their direct, immunological effects. We observed that for rotavirus vaccination in the US, the individual and population-level effects are considerable and complex. First, rotavirus vaccination led to a 95\% reduction in RVGE hospitalizations among vaccinated $0-4$ year olds. Second, introduction of the vaccine provided $35-60 \%$ protection against RVGE hospitalizations to unvaccinated individuals across age groups; this protection was generally limited to even post-vaccine years. Parallel patterns in indirect effects observed across all ages highlight the underrecognized burden of rotavirus outside the pediatric age range and emphasize the importance of infants in disease transmission. Lastly and surprisingly, estimates of direct VE varied annually, but we demonstrated that this observation is consistent with biases resulting from ICD-9/10 misclassification combined with biennial incidence patterns rather than variable vaccine performance. 

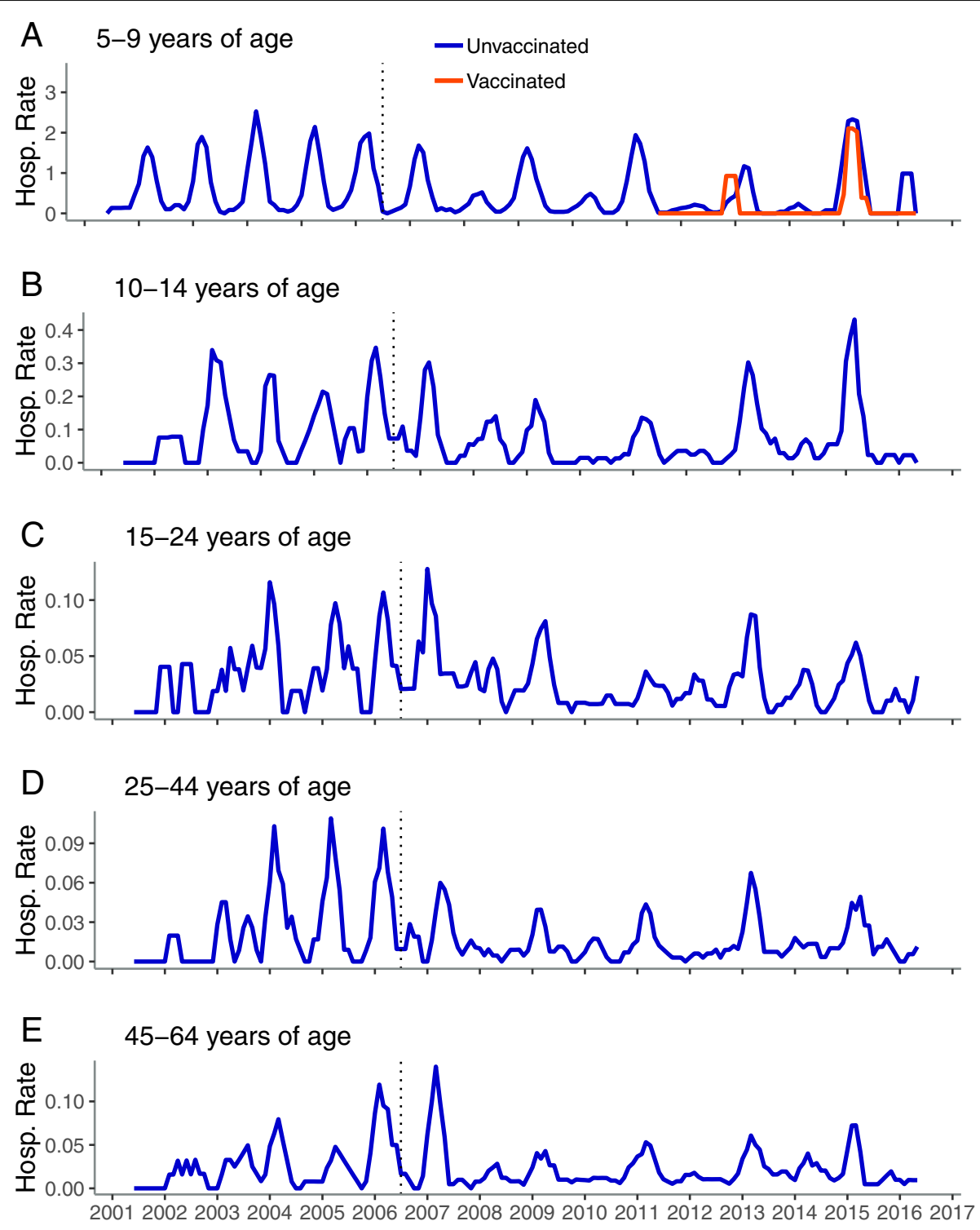

Fig. 2 Monthly inpatient RVGE rates per 10,000 person-years by age group, United States, July 2001-June 2016. . Legend: a: 5-9 years of age, b: 10-14 years of age, c: 15-24 years of age, $\mathbf{d}$ : 25-44 years of age, e: $45-64$ years of age ${ }^{\text {a }}$ Time series includes all years (including 2007 transition year) and all months (not restricted to the historic rotavirus season). Vertical dashed line represents July 2006 (time of vaccine introduction)

The decade-long post-vaccine period in the US provides a unique opportunity to assess the longer-term impacts of rotavirus vaccination across age groups and to quantify specific vaccine effects. A central strength of this study was the compilation and analysis of 15 years of national data from the MarketScan ${ }^{\bullet}$ Commercial Database. The large size, comprehensive information on vaccination status and consistent coding [20] enabled detailed analysis of nine years of post-vaccine data including age- and year-specific vaccine effects. Previous studies assessing the impacts of the rotavirus vaccine are limited to short-term post-vaccine periods, limited geographic ranges, or pediatric age groups $[14,19,23,24]$. This study contributes to existing literature on the effects of rotavirus vaccination across the age range [25] and is the first to estimate annual variation in vaccine effects over the nearly decade long post-vaccine period.

Overall, among all age groups combined, rotavirus hospitalization rates declined by nearly $70 \%$ after introduction of the vaccine. The youngest children were impacted most, however, the effects of the vaccine program were also felt outside the pediatric age range, bolstering existing evidence of indirect vaccine effects in the more immediate period following vaccine introduction $[11,25]$. Population-wide indirect benefits of infant vaccination were demonstrated by reductions in RVGE hospitalization 
Table 1 Vaccine effectiveness against RVGE hospitalization during the post-vaccine period by age group

\begin{tabular}{|c|c|c|c|c|}
\hline Age Group & Direct VE, \% (95\% Cl) & $\begin{array}{l}\text { Indirect VE, \% } \\
(95 \% \mathrm{CI})\end{array}$ & $\begin{array}{l}\text { Overall VE, \% } \\
(95 \% \text { CI) }\end{array}$ & $\begin{array}{l}\text { Total VE, \% } \\
(95 \% \text { CI) }\end{array}$ \\
\hline$<1$ & $80^{*}(70,87)$ & $79^{*}(66,87)$ & $88^{*}(82,92)$ & $96^{*}(93,97)$ \\
\hline 1 & $92^{*}(87,95)$ & $59^{*}(33,76)$ & $79^{*}(65,88)$ & $97^{*}(95,98)$ \\
\hline 2 & $87^{*}(76,93)$ & $43^{*}(4,67)$ & $68^{*}(44,83)$ & $93^{*}(86,96)$ \\
\hline 3 & $96^{*}(89,99)$ & $42(-2,68)$ & $71^{*}(47,84)$ & $97^{*}(93,99)$ \\
\hline 4 & $81^{*}(53,93)$ & $36(-25,68)$ & $59^{*}(22,79)$ & $88^{*}(70,96)$ \\
\hline $0-4$ & $87^{*}(83,90)$ & $60^{*}(48,69)$ & $78^{*}(71,83)$ & $95^{*}(93,96)$ \\
\hline $5-9$ & $47(-12,79)$ & $48^{*}(30,61)$ & $50^{*}(34,63)$ & $72^{*}(42,89)$ \\
\hline $10-14$ & & $46^{*}(14,67)$ & Equivalent to indirect $\mathrm{VE}^{\mathrm{a}}$ & \\
\hline $15-24$ & & $42^{*}(10,62)$ & & \\
\hline $25-44$ & & $56^{*}(36,70)$ & & \\
\hline $45-64$ & & $35^{*}(9,53)$ & & \\
\hline All ages & & & $69^{*}(62,76)$ & \\
\hline
\end{tabular}

*Represents significance at the alpha $=0.05$ level

andirect and overall VE are equivalent for children, adolescents, and adults over 9 years of age because there are no vaccinated individuals in these age groups

rates across age groups coupled with the emergence of biennial peaks in rates corresponding to those seen among vaccinated children. These findings reinforce the notion that infants are primary drivers of rotavirus infection across age groups. This theory is further supported by the increase in indirect benefits suggested among adults 2544 years of age, a population likely to have close contact with young children [26]. Yet, the general decline in indirect vaccine effects in older age groups indicate that rotavirus infection among these unvaccinated populations are not solely driven by infants.

The biennial pattern in disease incidence may have influenced our estimation of direct vaccine effectiveness. Imperfect classification results in a bias in vaccine effectiveness; if incidence is changing, the magnitude of the bias will vary because the number of cases to which the sensitivity and specificity of the codes are being applied changes. Indeed, we found that the annual variation in observed direct VE is entirely consistent with a vaccine with constant true effectiveness, imperfect sensitivity/ specificity of hospital coding, and varying incidence. In other words, variation in direct effectiveness may be due to the biennial patterns in disease incidence rather than true changes in vaccine effects. This bias could potentially arise in other estimates of direct vaccine effectiveness measured in the context of varying disease incidence and imperfect disease classification.

Cycles are a well-documented [11] feature of many infectious diseases. When host immunity combines with some seasonal factor (e.g. school terms or weather) season cycles may emerge [27]. Vaccination, which serves to reduce the number of individuals susceptible, may

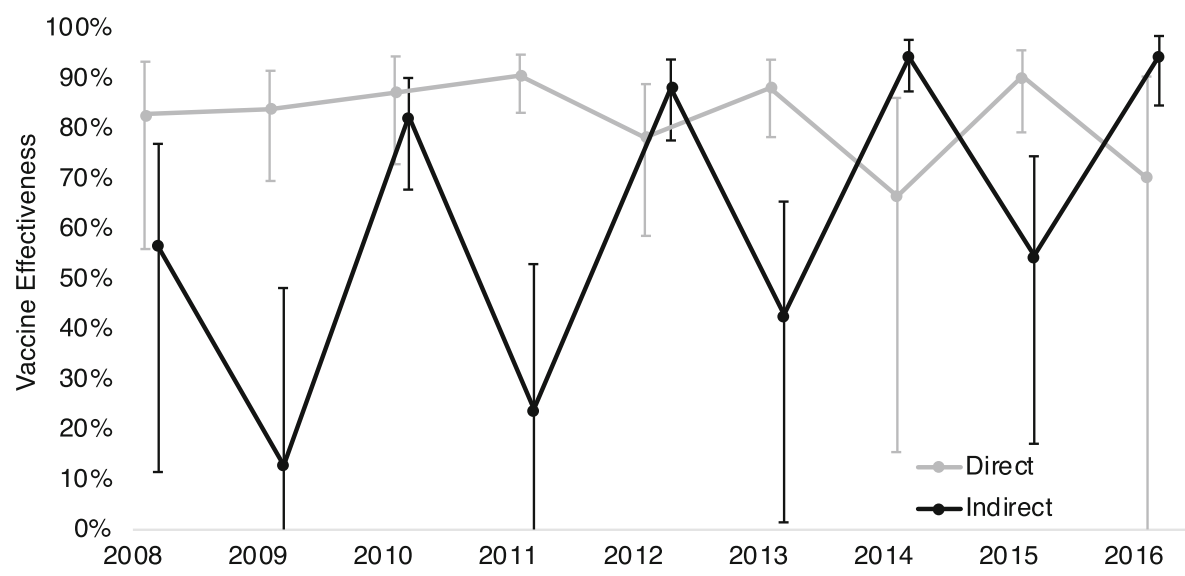

Fig. 3 Direct and indirect VE against RVGE by post-vaccine year, ${ }^{a}$ United States, children aged $0-4$ years ${ }^{b}$. Legend: ${ }^{a}$ Post-vaccine years defined as the

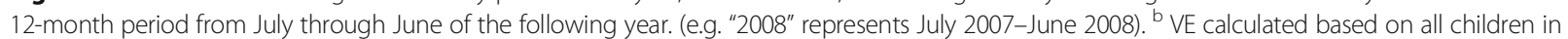
the age group, regardless of age eligibility for rotavirus vaccination, Bars represent 95\% confidence limits, Axis truncated at $0 \%$ 


\section{Color Key}
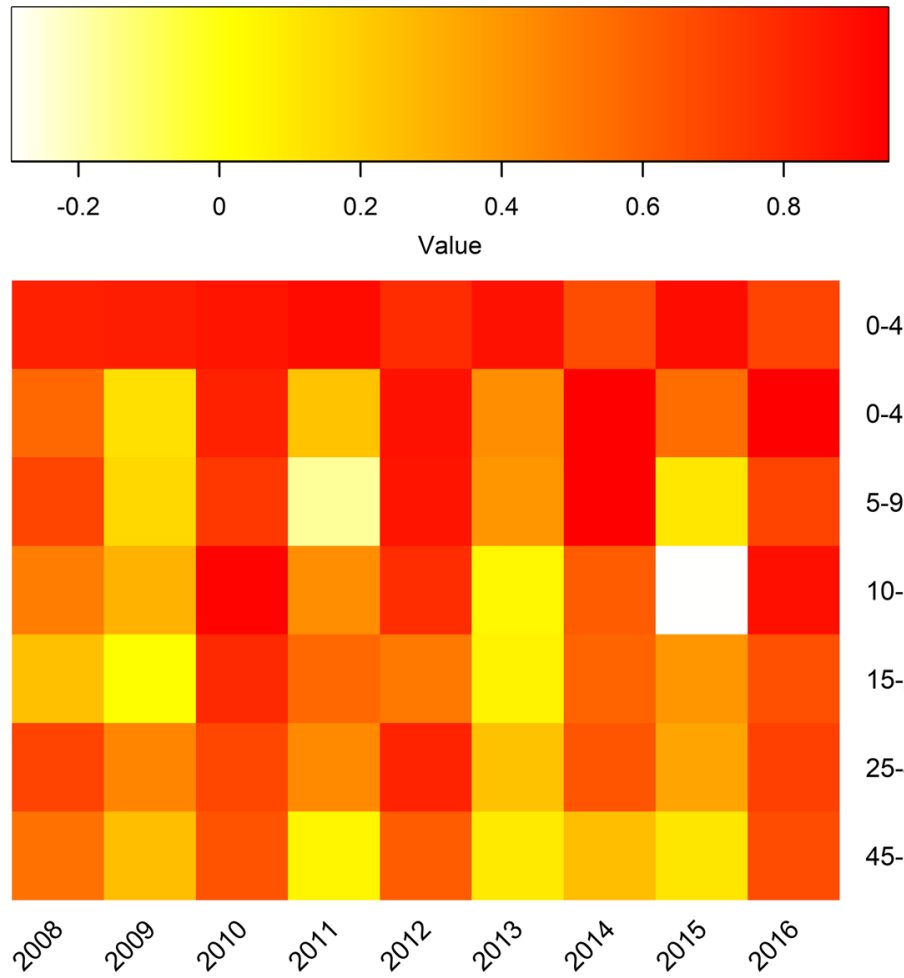

0-4 direct

0-4 indirect

$5-9$

$10-14$

$15-24$

$25-44$

$45-64$

Fig. 4 Direct and indirect VE against RVGE for each post-vaccine year, ${ }^{a}$ United States, by age group ${ }^{\mathrm{b}}$. Legend: ${ }^{a}$ Post-vaccine years defined as the 12-month period from July through June of the following year. (e.g. "2008" represents July 2007-June 2008). ' VE calculated based on all children in the age group, regardless of age eligibility for rotavirus vaccination

perturb these patterns and increase the inter-epidemic cycle. Indeed, this was predicted to occur for rotavirus under some epidemiological and vaccine-coverage scenarios [28-30]. Our study adds to the empirical data supporting this idea, but also extends it by documenting that these effects ripple across the age range. During even (low incidence) years, indirect VE was high across the age range while in odd (high incidence) years, there was little-to-no indirect VE.

Important limitations should be noted. We relied on ICD-9/10 codes which imperfectly capture RVGE. Not all individuals hospitalized for AGE are tested for rotavirus; evaluations among children during the pre-vaccine era have demonstrated that rotavirus ICD-9 coding has high specificity (97\%) and low sensitivity (less than 50\%) [12]. Little is known about the specificity and sensitivity of the coding in the post-vaccine era, possible misclassification or incomplete coding in the MarketScan ${ }^{\circ}$ Commercial Database, frequency of testing among adults, [31] or temporal changes in testing practices since vaccine introduction. One approach to address this limitation was to assess disease patterns in AGE rates, which have been shown to be valuable in assessing the burden of severe RVGE [13]. While we observed consistent patterns in RVGE and AGE in young age groups, vaccine impacts were not clear in older age groups, perhaps because an effect was

Table 2 Projected direct VE calculated in a hypothetical population compared with true and observed direct VE

\begin{tabular}{llllllll}
\hline Direct VE & $\begin{array}{l}2010 \%, \\
(95 \% \mathrm{Cl})\end{array}$ & $\begin{array}{l}2011 \%, \\
(95 \% \mathrm{Cl})\end{array}$ & $\begin{array}{l}2012 \%, \\
(95 \% \mathrm{Cl})\end{array}$ & $\begin{array}{l}2013 \%, \\
(95 \% \mathrm{Cl})\end{array}$ & $\begin{array}{l}2014 \%, \\
(95 \% \mathrm{Cl})\end{array}$ & $\begin{array}{l}2015 \%, \\
(95 \% \mathrm{Cl})\end{array}$ & $\begin{array}{l}2016 \%, \\
(95 \% \mathrm{Cl})\end{array}$ \\
\hline True $^{\mathrm{a}}$ & $95(90,98)$ & $95(93,97)$ & $95(87,98)$ & $95(92,97)$ & $95(82,99)$ & $95(92,97)$ & $95(80,99)$ \\
Observed $^{b}$ & $87(73,94)$ & $91(83,95)$ & $78(59,89)$ & $88(78,94)$ & $67(15,86)$ & $90(79,96)$ & $70(-10,90)$ \\
Projected $^{c}$ & $84(71,91)$ & $91(87,94)$ & $77(56,88)$ & $90(84,94)$ & $67(35,83)$ & $89(82,93)$ & $64(29,82)$ \\
\hline
\end{tabular}

${ }^{a}$ True VE is a set value used in the calculations

${ }^{b}$ Observed VE is direct VE estimated using the MarketScan ${ }^{\circledR}$ Commercial Database data

'Projected VE is the direct VE calculated in a hypothetical population of 1,000,000 with vaccine coverage of $50 \%$, true VE of $95 \%$, sensitivity of 0.5 , specificity of 0.99, and estimated RVGE incidence among unvaccinated children based on the MarketScan ${ }^{\oplus}$ data for each year from 2010 to 2016 

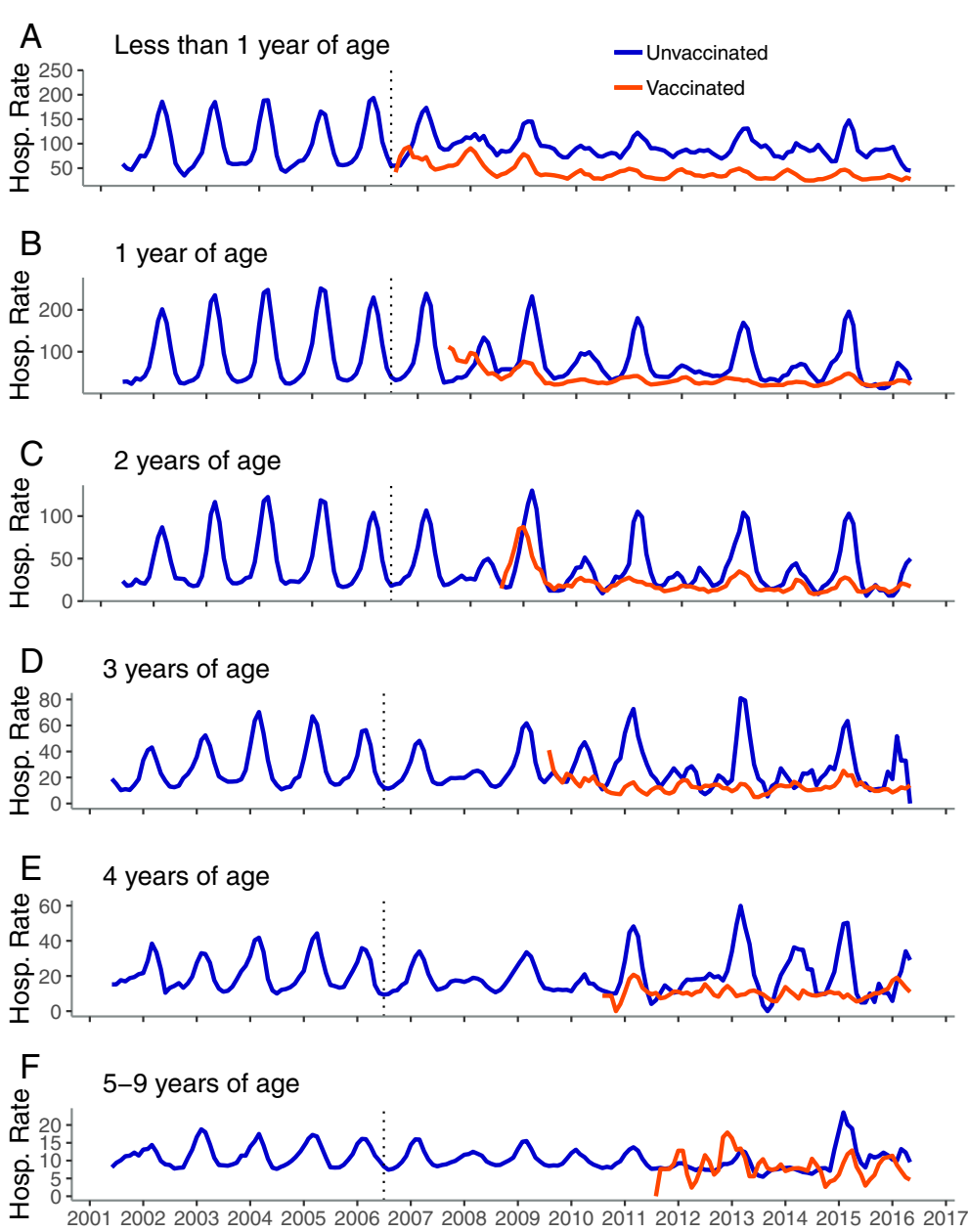

Fig. 5 Time series of monthly inpatient AGE rates per 10,000 person-years, United States, July 2001-June 2016. . Legend: a: less than 1 year of age, b: 1 year of age, c: 2 years of age, $\mathbf{d}$ : 3 years of age, e: 4 years of age, f: 5-9 years of age ${ }^{\text {a }}$ Timeseries includes all years (including 2007 transition year) and all months (not restricted to the historic rotavirus season). Vertical dashed line represents July 2006 (time of vaccine introduction)

overwhelmed by an increasing secular trend in AGE among older individuals (see additional materials).

A primary concern in analysis of time series data is time varying confounders. We aimed to adjust for potential unknown temporal trends by testing the sensitivity of all models to the inclusion of a sequential time variable; none of the RVGE models were found to be sensitive to this variable. National coverage levels for the rotavirus vaccine increased in the years immediately following its introduction though have plateaued around $73 \%$ since 2013 [11, 32]; coverage may indirectly contribute to the variation in direct and indirect effectiveness observed by impacting the number of susceptibles in the population. There is evidence of changes in prevalence of circulating rotavirus strains in the US since vaccine introduction though no consistent pattern has been observed [33], making this unlikely to be the driver of the distinct patterns observed for RVGE rates and VE. It is possible that increased frequency of testing [34] and improved laboratory techniques [35] may impact the number of RVGE cases over the post-vaccine period. If these changes have occurred, they would likely result in an underestimation of the VE measures. Finally, this study may have limited generalizability as the data used did not include the under-insured, individuals on Medicaid, and individuals aged 65 years and older. We are unable to draw conclusions about specific patterns of illness or VE among populations not included in the dataset, such as the underinsured who may have different levels of vaccine coverage [36]. Nonetheless, the effects observed are a function of the wider US population, not just those captured in the dataset.

This study provides new evidence of the individual and population-wide impacts of the rotavirus vaccine and highlights an important potential for bias in direct VE estimation, not previously investigated for rotavirus vaccination. Measurements of direct rotavirus VE may be prone to downward bias in the post-vaccine era due to reductions in disease incidence resulting in lower and 
Table $\mathbf{3}$ Vaccine effectiveness against AGE hospitalization during the post-vaccine period by age group

\begin{tabular}{lllll}
\hline Age Group & $\begin{array}{l}\text { Direct VE, \% } \\
(95 \% \mathrm{Cl})\end{array}$ & $\begin{array}{l}\text { Indirect VE, \% } \\
(95 \% \mathrm{Cl})\end{array}$ & $\begin{array}{l}\text { Overall VE, \% } \\
(95 \% \mathrm{Cl})\end{array}$ & $\begin{array}{l}\text { Total VE, \% } \\
(95 \% \mathrm{Cl})\end{array}$ \\
\hline$<1$ & $59^{*}(54,64)$ & $25^{*}(12,36)$ & $49^{*}(37,58)$ & $69^{*}(63,74)$ \\
1 & $63^{*}(55,69)$ & $38^{*}(20,52)$ & $59^{*}(46,68)$ & $77^{*}(71,81)$ \\
2 & $57^{*}(44,66)$ & $28^{*}(5,46)$ & $49^{*}(33,62)$ & $69^{*}(59,76)$ \\
3 & $60^{*}(49,68)$ & $16(-7,34)$ & $42^{*}(27,55)$ & $67^{*}(58,74)$ \\
4 & $54^{*}(42,64)$ & $11(-13,29)$ & $33^{*}(16,46)$ & $60^{*}(47,70)$ \\
$0-4$ & $56^{*}(50,61)$ & $27^{*}(15,38)$ & $48^{*}(40,55)$ & $68^{*}(63,72)$ \\
$5-9$ & $33^{*}(19,45)$ & $16^{*}(8,24)$ & $20^{*}(12,27)$ & $44^{*}(31,55)$ \\
\hline
\end{tabular}

${ }^{*}$ Represents significance at the alpha $=0.05$ level

changing predictive value of diagnosis. A vaccinated child in the post-vaccine era has a 95\% reduced risk of RVGE hospitalization compared to a child in the prevaccine era. Vaccine benefits extended to unvaccinated individuals across the age range and demonstrate the important role of infants in rotavirus transmission.

\section{Conclusions}

This comprehensive estimation of the range of vaccine effects provides new evidence of the individual and population-wide impacts of infant rotavirus vaccination and highlights an important potential for bias in direct vaccine effectiveness estimation. Our findings demonstrate the high direct effectiveness of infant rotavirus vaccination and suggest that the impacts of the vaccine program can be felt population-wide, including among adults and unvaccinated children. A novel finding was that imperfect disease classification combined with changing disease incidence during the post-vaccine period may lead to downward bias in the estimated direct vaccine effectiveness. This bias should be considered in other estimates of direct vaccine effectiveness in the context of varying disease incidence and imperfect case classification.

\section{Additional file}

Additional file 1: Table S1. Number of RVGE and AGE cases by age group, United States, July 2001-June 2016. ${ }^{\text {a }}$. Figure S1. Time series of monthly inpatient AGE rates per 10,000 person-years by age group, United States, July 2001-June 2016. ${ }^{\text {. }}$ (DOCX 20584 kb)

\section{Abbreviations}

AGE: All-cause acute gastroenteritis; AIC: Akaike information criterion; Cl: Confidence interval; CPT: Current Procedural Terminology; ICD-10: International Classification of Diseases, Tenth Revision; ICD-9: International Classification of Diseases, Ninth Revision; RR: Rate ratio; RV1: Rotarix; RV5: RotaTeq; RVGE: Rotavirus gastroenteritis; US: United States; VE: Vaccine effectiveness

\section{Acknowledgements}

Not applicable.

\section{Funding}

This work received no dedicated funding.

\section{Availability of data and materials}

The data that support the findings of this study are available from the $\mathrm{IBM}^{\circledast}$ MarketScan ${ }^{\oplus}$ Research Databases but restrictions apply to the availability of these data, which were used under license for the current study, and so are not publicly available. Data are however available from the authors upon reasonable request and with permission from $\mathrm{IBM}^{\oplus}$ Watson Health ${ }^{\mathrm{TM}}$.

\section{Authors' contributions}

The study was conceptualized by BAL with input from JMB and RMD and supervisory support from UDP. RMD designed and conducted data extraction from the $\mathrm{IBM}^{\oplus}$ MarketScan ${ }^{\oplus}$ Research Databases with input from $J M B$ and BAL. JMB led the main study analysis with support from BAL. JC, $J M B$ and $B A L$ conducted the sub-analysis examining variations in direct vaccine effects. JMB drafted the manuscript with supervisory input from BAL. All authors reviewed, edited, and approved the manuscript.

\section{Ethics approval and consent to participate}

This study involved de-identified, aggregate data and was not subject to Institutional Review Board approval at Emory University or the Centers for Disease Control and Prevention.

\section{Consent for publication}

Not applicable.

\section{Competing interests}

BAL has received personal fees from Takeda Pharmaceuticals for service on their Norovirus Advisory Board outside the submitted work. JMB, RMD, JC and UDP have no conflicts of interest.

\section{Publisher's Note}

Springer Nature remains neutral with regard to jurisdictional claims in published maps and institutional affiliations.

\section{Author details}

${ }^{1}$ Department of Epidemiology, Rollins School of Public Health, Emory University, 1518 Clifton Road NE, Atlanta, GA 30322, USA. Division of Viral Diseases, National Center for Immunization and Respiratory Diseases, Centers for Disease Control and Prevention, 1600 Clifton Road NE, Atlanta, GA 30333, USA. ${ }^{3}$ MAXIMUS Federal, contracting agency to the Division of Viral Diseases, National Center for Immunization and Respiratory Diseases, Centers for Disease Control and Prevention, 1600 Clifton Road NE, Atlanta, GA 30333, USA.

Received: 2 November 2018 Accepted: 13 February 2019 Published online: 22 February 2019

\section{References}

1. International Vaccine Access Center (IVAC). VIEW-hub Report: Global Vaccine Introduction and Implementation. Baltimore: Johns Hopkins Bloomberg School of Public Health; 2016.

2. Tate JE, Panozzo CA, Payne DC, Patel MM, Cortese MM, Fowlkes AL, et al. Decline and change in seasonality of US rotavirus activity after the introduction of rotavirus vaccine. PEDIATRICS. 2009;124(2):465-71.

3. Parashar UD, Alexander JP, Glass RI. Advisory Committee on Immunization Practices (ACIP), Centers for Disease Control and Prevention (CDC). Prevention of rotavirus gastroenteritis among infants and children. Recommendations of the Advisory Committee on Immunization Practices (ACIP). MMWR Recomm Rep Morb Mortal Wkly Rep Recomm Rep. 2006; 55(RR-12):1-13.

4. Charles MD, Holman RC, Curns AT, Parashar UD, Glass RI, Bresee JS. Hospitalizations Associated With Rotavirus Gastroenteritis in the United States, 1993-2002. Pediatr Infect Dis J. 2006;25(6):489-93.

5. Tate JE, Haynes A, Payne DC, Cortese MM, Lopman BA, Patel MM, et al. Trends in National Rotavirus Activity Before and After Introduction of Rotavirus Vaccine into the National Immunization Program in the United States, 2000 to 2012. Pediatr Infect Dis J. 2013;32(7):741-4.

6. Leshem E, Tate JE, Steiner CA, Curns AT, Lopman BA, Parashar UD. Acute gastroenteritis hospitalizations among US children following implementation of the rotavirus vaccine. JAMA. 2015;313(22):2282.

7. Hemming-Harlo M, Markkula J, Huhti L, Salminen M, Vesikari T. Decrease of rotavirus gastroenteritis to a low level without resurgence for five years after 
universal RotaTeq vaccination in Finland. Pediatr Infect Dis J. 2016;35(12): 1304-8.

8. Sabbe M, Berger N, Blommaert A, Ogunjimi B, Grammens T, Callens M, et al. Sustained low rotavirus activity and hospitalisation rates in the postvaccination era in Belgium, 2007 to 2014. Eurosurveillance. 2016 [cited 2018 Jul 16];21(27). Available from: https://www.ncbi.nlm.nih.gov/pubmed/ 27418466

9. Jayasinghe S, Macartney K. Estimating rotavirus gastroenteritis hospitalisations by using hospital episode statistics before and after the introduction of rotavirus vaccine in Australia. Vaccine. 2013;31(6):967-72

10. Rha B, Tate JE, Payne DC, Cortese MM, Lopman BA, Curns AT, et al. Effectiveness and impact of rotavirus vaccines in the United States - 2006-2012. Expert Rev Vaccines. 2014;13(3):365-76.

11. Pindyck T, Tate JE, Parashar UD. A decade of experience with rotavirus vaccination in the United States - vaccine uptake, effectiveness, and impact. Expert Rev Vaccines. 2018;17(7):593-606.

12. Hsu VP, Staat MA, Roberts N, Thieman C, Bernstein DI, Bresee J, et al. Use of active surveillance to validate International Classification of Diseases code estimates of rotavirus hospitalizations in children. Pediatrics. 2005;115(1):78-82.

13. Patel MM, Tate JE, Selvarangan R, Daskalaki I, Jackson MA, Curns AT, et al. Routine Laboratory Testing Data for Surveillance of Rotavirus Hospitalizations to Evaluate the Impact of Vaccination. Pediatr Infect Dis J. 2007;26(10):914-9.

14. Lopman BA, Curns AT, Yen C, Parashar UD. Infant rotavirus vaccination may provide indirect protection to older children and adults in the United States. J Infect Dis. 2011;204(7):980-6.

15. Halloran ME, Struchiner CJ, Longini IM. Study designs for evaluating different efficacy and effectiveness aspects of vaccines. Am J Epidemiol. 1997;146(10):789-803.

16. Haber M. Estimation of the direct and indirect effects of vaccination. Stat Med. 1999;18(16):2101-9.

17. Smith PG, Rodrigues LC, Fine PE. Assessment of the protective efficacy of vaccines against common diseases using case-control and cohort studies. Int J Epidemiol. 1984;13(1):87-93.

18. Goldstein E, Pitzer VE, O'Hagan JJ, Lipsitch M. Temporally varying relative risks for infectious diseases: implications for infectious disease control. Epidemiology. 2017;28(1):136-44.

19. Panozzo CA, Becker-Dreps S, Pate V, Weber DJ, Jonsson Funk M, Sturmer T, et al. Direct, indirect, Total, and overall effectiveness of the rotavirus vaccines for the prevention of gastroenteritis hospitalizations in privately insured US children, 2007-2010. Am J Epidemiol. 2014;179(7):895-909.

20. Hansen LG. Health Research Data for the Real World: The Marketscan Databases [Internet]. Truven Health Analytics; 2011 [cited 2018 Aug 16]. Available from: http://truvenhealth.com/portals/0/assets/PH_11238_0612_ TEMP_MarketScan_WP_FINAL.pdf

21. Leshem E, Moritz RE, Curns AT, Zhou F, Tate JE, Lopman BA, et al. Rotavirus vaccines and health care utilization for diarrhea in the United States (20072011). Pediatrics. 2014;134(1):15-23

22. Centers for Disease Control and Prevention, Department of Health and Human Services. The Recommended Immunization Schedules for Psersons Aged 0 Through 18 Years, United States, 2016 [Internet]. [cited 2019 Jan 17]. Available from: https://www.cdc.gov/vaccines/schedules/downloads/past/ 2016-child.pdf

23. Payne DC, Staat MA, Edwards KM, Szilagyi PG, Weinberg GA, Hall CB, et al. Direct and indirect effects of rotavirus vaccination upon childhood hospitalizations in 3 US counties, 2006-2009. Clin Infect Dis. 2011;53(3):245-53.

24. Cortes JE, Curns AT, Tate JE, Cortese MM, Patel MM, Zhou F, et al. Rotavirus vaccine and health care utilization for diarrhea in U.S. children. N Engl J Med. 2011;365(12):1108-17.

25. Baker JM, Tate JE, Steiner CA, Haber MJ, Parashar UD, Lopman BA. Longerterm direct and indirect effects of infant rotavirus vaccination across all ages in the US; 2000-2013: analysis of a large hospital discharge dataset. Clin Infect Dis [Internet]. 2018 [cited 2018 Jul 19]; Available from: https:// academic.oup.com/cid/advance-article/doi/10.1093/cid/ciy580/5055445

26. Mossong J, Hens N, Jit M, Beutels P, Auranen K, Mikolajczyk R, et al. Social Contacts and Mixing Patterns Relevant to the Spread of Infectious Diseases. Riley S, editor. PLoS Med. 2008 Mar 25;5(3):e74

27. Grassly NC, Fraser C. Seasonal infectious disease epidemiology. Proc R Soc B Biol Sci. 2006;273(1600):2541-50.

28. Pitzer VE, Atkins KE, de Blasio BF, Van Effelterre T, Atchison CJ, Harris JP, et al. Direct and Indirect Effects of Rotavirus Vaccination: Comparing
Predictions from Transmission Dynamic Models. Roberts MG, editor. PLoS ONE. 2012;7(8):e42320.

29. Pitzer VE, Viboud C, Simonsen L, Steiner C, Panozzo CA, Alonso WJ, et al. Demographic variability, vaccination, and the spatiotemporal dynamics of rotavirus epidemics. Science. 2009;325(5938):290-4.

30. Atchison C, Lopman B, Edmunds WJ. Modelling the seasonality of rotavirus disease and the impact of vaccination in England and Wales. Vaccine. 2010;28(18):3118-26.

31. Anderson EJ, Weber SG. Rotavirus infection in adults. Lancet Infect Dis. 2004:4(2):91-9.

32. Hill HA, Elam-Evans LD, Yankey D, Singleton JA, Kang Y. Vaccination coverage among children aged 19-35 months — United States, 2016. MMWR Morb Mortal Wkly Rep. 2017;66(43):1171-7.

33. Bowen MD, Mijatovic-Rustempasic S, Esona MD, Teel EN, Gautam R, Sturgeon $\mathrm{M}$, et al. Rotavirus strain trends during the Postlicensure vaccine era: United States, 2008-2013. J Infect Dis. 2016;214(5):732-8.

34. Aliabadi N, Tate JE, Haynes AK, Parashar UD. Centers for Disease Control and Prevention (CDC). Sustained decrease in laboratory detection of rotavirus after implementation of routine vaccination-United States, 2000-2014. MMWR Morb Mortal Wkly Rep. 2015;64(13):337-42.

35. Tate JE, Mijatovic-Rustempasic S, Tam Kl, Lyde FC, Payne DC, Szilagyi P, et al. Comparison of 2 assays for diagnosing rotavirus and evaluating vaccine effectiveness in children with gastroenteritis. Emerg Infect Dis. 2013;19(8): 1245-52.

36. Hill HA, Elam-Evans LD, Yankey D, Singleton JA, Dietz V. Vaccination coverage among children aged 19-35 months - United States, 2015. MMWR Morb Mortal Wkly Rep. 2016;65(39):1065-71.
Ready to submit your research? Choose BMC and benefit from:

- fast, convenient online submission

- thorough peer review by experienced researchers in your field

- rapid publication on acceptance

- support for research data, including large and complex data types

- gold Open Access which fosters wider collaboration and increased citations

- maximum visibility for your research: over $100 \mathrm{M}$ website views per year

At BMC, research is always in progress.

Learn more biomedcentral.com/submissions 